\title{
ON APPLYING MATHEMATICS: REFLECTIONS AND EXAMPLES
}

\author{
BY \\ M. KAC \\ Rockefeller University
}

1. I have felt for a long time that in discussing the perennial topic of the role of applied mathematics one is inclined to overlook that at the heart of the matter is the subtle and fundamental question of the relation of mathematics to non-mathematical disciplines.

We tend to think of "applied" as being roughly synonymous with "useful" or "practical", but I should like to argue that applying mathematics is an activity which often transcends pragmatic considerations and that we should also be concerned with a deeper exploration of what this activity is or ought to be.

As usual, it is easier to say what it ought not to be, and here I cannot resist referring once more to a wartime cartoon depicting two chemists surveying ruefully a little pile of sand amidst complex and impressive-looking apparatus. The caption read: "Nobody really wanted a dehydrated elephant but it is nice to see what can be done". I am sure that we can all agree that applying mathematics should not result in creation of "dehydrated elephants".

2. But how can one tell?

A necessary (though by no means sufficient) prerequisite is to know the subject or discipline to which we apply mathematics and to know it with such intimacy as to understand its spirit and to appreciate its lore.

When we propose to apply mathematics we are stepping outside our own realm, and such a venture is not without dangers. For having stepped out, we must be prepared to be judged by standards not of our own making and to play games whose rules have been laid down with little or no consultation with us.

Of course, we don't have to play, but if we do we have to abide by the rules and above all not try to change them merely because we find them uncomfortable or restrictive.

3. From now on let me stick to physics, not only because it is the only non-mathematical discipline with which I am reasonably familiar but also because physics and mathematics have had close ties in the past and because after a period of alienation there are signs of a rapprochement. My joy at the prospect of the two great disciplines coming together again is somewhat mitigated by the fear of "dehydrated elephants" on one side facing a rigid and even hostile pragmatism on the other.

Since there is nothing I can do about pragmatism of physics (and some physicists!) let me limit my discussion to exploring some of the ways in which mathematics can be significantly applied to certain problems of physics. Let me also state that I speak only for myself and that I do not expect my views to be universally accepted. Quite to the contrary, I expect opposition, and would welcome a debate or even a confrontation. 
4. First, it seems self-evident that mathematics is not likely to be much help in discovering laws of nature. If a mathematician wants to make a contribution on this (and I admit it is the highest) level, he will have to master so much experimental material and train himself to think in a way so different from the one he has been accustomed to that he will, in effect, cease to be a mathematician. ${ }^{1}$ Perhaps it is well to be reminded by the way of analogy that while in recent years a number of physicists have made significant contributions to biology they accomplished this not because they were physicists but because they became biologists. I cannot, of course, claim that their training in physics had nothing to do with their successes in biology. More likely it has a great deal to do with it, and by the same token a mathematician who becomes a physicist (or a biologist, or an economist) may benefit greatly from his training as a mathematician, but this is not what I am concerned with today.

As mathematicians we come in when this or that law of nature has been discovered, and our role is usually twofold: (a) to help find ways in which the law can "best" be formulated and (b) to help in drawing conclusions, which hopefully will be significant to the further development of the subject. Of the two, the first role is fraught with more danger, since it tends to provide fertile ground for breeding "dehydrated elephants". This is especially true today because contemporary mathematics is geared to playing with "formulations" and physics, so full of vague, unprecise and unrigorous statements, seems to cry out for being better "formulated" and fitted into familiar structures.

In a recent article "Hamiltonian mechanics and geometry" [1], Professor Saunders MacLane, a leader of and an eloquent spokesman for the strongly abstract trend in mathematics, says: "Just as each generation of historians must analyse the past again, so in the exact sciences we must in each period take up the renewed struggle to present as clearly as we can the underlying ideas of mathematics". A little farther he continues: "Many cumbersome developments in the standard treatments of mechanics can be simplified and better understood when formulated with modern conceptual tools, as in the well-known case of the use of the 'universal' definition of tensor products of vector spaces to simplify some of the notational excesses of tensor analysis as traditionally used in relativity theory".

I was groping for a suitable rebuttal to these views when help can from an unexpected source. In a review of a book On dynamical systems in biology [2], a biologist, Joel E. Cohen wrote:

When somebody else has done the dirty tedious work of showing that a mathematically formulated physical principle leads to predictions correct to a specified number of decimal

1 To gain an insight into the kind of thinking that often goes into making a physical discovery, I strongly recommend Chapter 10 ("The Essential Nature of the Quantum Hypothesis") of M. J. Klein's superb biography of Paul Ehrenfest (Paul Ehrenfest, North Holland Publishing Co.-American Elsevier Publishing Co., 1970). It concerns the discovery by Planck of his famous radiation law and the reverberations it produced in the foundations of statistical physics. It is instructive to recall that it was not any kind of logical necessity that was the motivating force behind the development of the new ideas. In fact, to use a modern expression, logic was not in it. What kept the thing going, in spite of it being a veritable morass of contradictions and obscurities, was a profound respect for experiment and an infinite faith in the second law. Only after predictions of the new theory had been spectacularly confirmed and verified did Poincare (and Ehrenfest independently and somewhat earlier) attempt (in 1911 and 1912) a systematic and logical analysis of the foundations of the new theory. In vain, as it turned out, for twenty-five years later quantum mechanics rendered most (though by no means all!) of it obsolete. 
places in the boring world of Euclidean 3-space with Cartesian coordinates, theoreticians and textbook writers can axiomatize, generalize and dazzle your eyes with the most coordinate-free cosmically invariant representations you please.

This brief (and somewhat angry) paragraph contains the pragmatist's case against some of the "games mathematicians like to play". In cruder language it amounts to saying: don't dazzle me with this elegant stuff-tell me something I didn't know.

5. Still, it would be narrow-minded and unjust to discourage searches for new formulations. No one could deny that Minkowski's formulation of the special theory of relativity has been decisive in the development of this theory, and although most of us (including myself) would not take "notational excesses of tensor analysis" as seriously as Professor MacLane seems to take them, it is beyond dispute that Riemannian geometry is the proper setting for Einstein's general theory. But one would find much less agreement as to the value of the subtle and elegant axiomatization of thermodynamics by Caratheodory, and one could cite examples of reformulations whose pertinence and value is even more in doubt.

It is not quite easy to explain why "geometrization" of relativity theory (both special and general) is among the most glorious applications of mathematics while Caratheodory's reformulation is a bit of a "dehydrated elephant", but it must have something to do with the distinction between facing up to problems which are, so to speak, God-given and those which are only man-made.

If I understand Professor MacLane correctly, he would like us to keep reexamining physics in the light of conceptual advances in mathematics. Without proper checks, this would, in my opinion, constitute the broadest license for creation of "dehydrated elephants". It would also add to a kind of self-delusion that one is contributing to a subject while in reality all one is accomplishing is discovering that all the time one has been speaking prose.

As a reliable check, I would propose testing formulations against some of the questions (preferably hitherto unanswered) which this or that field evolved over a period of time. If a dent (even a small one!) can be made, the formulation has shown viability and should be pursued. If, however, all that comes out is the same old stuff in a different guise-well, write it off as self-education, put it in a notebook and above all don't feel compelled to publish it.

6. So much for the reflections. In my title I also promised examples, and I shall now present one which will illustrate what I have in mind. It is taken from the field of statistical mechanics which in recent years has been attracting quite a bit of attention from mathematicians. Although I shall stick mainly to classical theory, let me begin by giving the quantum-mechanical background of the problems to be discussed.

To explain the phenomenon of ferromagnetism Heisenberg proposed the following model. Consider a three-dimensional lattice (crystal) and assume that the atom on each site $\mathbf{R}$ acts as a little magnet. Let the Hamiltonian of the interaction between these magnets be assumed to be of the form

$$
H_{\mathrm{int}}=-\sum_{\mathbf{R}, \mathbf{R}^{\prime}} J\left(\mathbf{R}-\mathbf{R}^{\prime}\right) \mathbf{\sigma}(\mathbf{R}) \cdot \boldsymbol{\sigma}\left(\mathbf{R}^{\prime}\right),
$$

where

$$
\mathbf{\sigma}(\mathbf{R}) \cdot \boldsymbol{\sigma}\left(\mathbf{R}^{\prime}\right)=\sigma_{x}(\mathbf{R}) \sigma_{x}\left(\mathbf{R}^{\prime}\right)+\sigma_{\nu}(\mathbf{R}) \sigma_{\nu}\left(\mathbf{R}^{\prime}\right)+\sigma_{z}(\mathbf{R}) \sigma_{z}\left(\mathbf{R}^{\prime}\right)
$$


and $\sigma_{x}, \sigma_{y}, \sigma_{z}$ are the Pauli matrices. When associated with different sites, they are assumed to commute, e.g. $\sigma_{x}(\mathbf{R}) \sigma_{y}\left(\mathbf{R}^{\prime}\right)=\sigma_{y}\left(\mathbf{R}^{\prime}\right) \sigma_{x}(\mathbf{R}), \mathbf{R} \neq \mathbf{R}^{\prime}$, while, of course, on the same site they obey the familiar commutation relations.

If placed in a magnetic field in the $z$-direction, say, one has to add an external Hamiltonian of the form $H_{\text {ext }}=-a \sum_{\mathrm{R}} \sigma_{z}(\mathrm{R})$, where $a$ measures the strength of the external field.

The Hamiltonian is thus

$$
H=H_{\mathrm{int}}+H_{\mathrm{ext}}=-\sum_{\mathbf{R}, \mathbf{R}^{\prime}} J\left(\mathrm{R}-\mathrm{R}^{\prime}\right) \boldsymbol{\sigma}(\mathrm{R}) \cdot \boldsymbol{\sigma}\left(\mathrm{R}^{\prime}\right)-a \sum_{\mathbf{R}} \sigma_{z}(\mathrm{R})
$$

and to have any hope of ferromagnetic behavior, one must assume that $J(\mathbf{R}) \geq 0$. The simplest non-trivial case is when $J(\mathbf{R})=0$, except when $\mathbf{R}$ is one of the "unit" vectors which generate the lattice (the case of nearest-neighbor interaction).

If the system as described above is in equilibrium with a heat bath of absolute temperature $T$, then according to the rules of quantum statistical mechanics (which I shall accept without question) the magnetization $m$ is given by the formula

$$
m=\frac{\operatorname{Trace}\left\{\frac{1}{N}\left(\sum \sigma_{z}(\mathrm{R})\right) \exp (-\beta H)\right\}}{\text { Trace }\{\exp (-\beta H)\}}
$$

where $\beta=1 / \kappa T$, and $\kappa$ is the Boltzmann constant.

As defined, $m$ still depends on the size of the lattice and one must first take the socalled thermodynamic limit, i.e. the limit as the size of the lattice becomes infinite. Assuming a cubic lattice of $\sqrt[3]{N} \times \sqrt[3]{N} \times \sqrt[3]{N}=N$ points, the thermodynamic limit amounts to letting $N \rightarrow \infty$.

Set

$$
m^{*}(\omega ; \beta)=\lim _{N \rightarrow \infty} m
$$

where $\omega=a \beta=a / \kappa T$ and it is $m^{*}$ that is of interest.

The problem is to prove that there exists a critical temperature $T_{c}$ (or equivalently a critical $\beta_{c}$ ) such that

$$
\lim _{\omega \rightarrow 0} m^{*}(\omega ; \beta)=0 \quad \beta<\beta_{c}
$$

and

$$
\lim _{\omega \rightarrow 0+} m^{*}(\omega ; \beta) \neq 0 \quad \beta>\beta_{c} .
$$

In other words, we must prove that for sufficiently low temperatures there will be (residual) magnetization even when the external field is removed $(\omega \rightarrow 0)$.

7. Although the problem is roughly forty-five years old and has attracted a great deal of attention it remains unsolved. Even to prove that $m^{*}$ is well-defined (i.e. that the thermodynamic limit exists) is not easy, although it is also the least exciting part.

Physicists too sometimes trade realism for solubility, and the Heisenberg model has been subjected to various simplifications. The most celebrated consists in replacing $\boldsymbol{\sigma}(\mathbf{R}) \cdot \boldsymbol{\sigma}\left(\mathbf{R}^{\prime}\right)$ by $\sigma_{z}(\mathbf{R}) \sigma_{z}\left(\mathbf{R}^{\prime}\right)$, which removes all the difficulties connected with noncommutativity. The resulting model is known as the Ising model, though it was apparently first suggested by Lenz. The formula for $m$ is now quite elementary (owing to 
the fact that $\sigma_{\mathbf{z}}(\mathbf{R})$ is a $2 \times 2$ matrix with eigenvalues +1 and -1 ), namely

$$
m=\frac{\sum \frac{1}{N}\left(\sum \mu(\mathrm{R})\right) \exp \left\{\beta \sum J\left(\mathrm{R}-\mathrm{R}^{\prime}\right) \mu(\mathrm{R}) \mu\left(\mathrm{R}^{\prime}\right)+\omega \sum \mu(\mathrm{R})\right\}}{\sum \exp \left\{\beta \left\llcornerJ\left(\mathrm{R}-\mathrm{R}^{\prime}\right) \mu(\mathrm{R}) \mu\left(\mathrm{R}^{\prime}\right)+\omega\llcorner\mu(\mathrm{R})\}\right.\right.},
$$

where each $\mu(\mathbf{R})$ can assume the value \pm 1 and the summations on the farthest left are over all the $2^{N}$ assignments of plus ones and minus ones to different sites of the lattice.

Again $m^{*}(\omega ; \beta)$ is defined as the thermodynamic limit of $m$ and the question is again whether for sufficiently low temperatures one has residual magnetization. This time the answer is in the affirmative even if the lattice is two-dimensional!

On the other hand, it was recently proved by Mermin and Wagner [3] that the Heisenberg model for a two-dimensional lattice has no residual magnetization at any temperature provided $J(R)$ is sufficiently short-ranged, e.g. nearest-neighbor.

The proper understanding of the role of dimensionality of the lattice is clearly not only of physical importance but also of considerable mathematical interest.

8. The question of the role of dimensionality appears also in a somewhat different setting.

There is a classical version of the Heisenberg model in which at each lattice site we have again a little magnet represented by an ordinary three-dimensional vector of unit length. The interaction Hamiltonian is assumed to be of the form

$$
H_{\mathrm{int}}=-\sum J\left(\mathbf{R}-\mathbf{R}^{\prime}\right) \boldsymbol{\sigma}(\mathbf{R}) \cdot \boldsymbol{\sigma}\left(\mathbf{R}^{\prime}\right)
$$

and the effect of the external magnetic field (again in the $z$-direction) is represented by the Hamiltonian

$$
H_{\text {ext }}=-a \sum \sigma_{z}(\mathbf{R}),
$$

where $\sigma_{z}(\mathbf{R})$ is simply the $z$-component of the vector $\boldsymbol{\delta}(\mathbf{R})$. It should also be noted that now the scalar product $\mathbf{\sigma}(\mathbf{R}) \cdot \boldsymbol{\sigma}\left(\mathbf{R}^{\prime}\right)$ is given by the standard formula

$$
\boldsymbol{\sigma}(\mathbf{R}) \cdot \boldsymbol{\sigma}\left(\mathbf{R}^{\prime}\right)=\sigma_{x}(\mathbf{R}) \sigma_{x}\left(\mathbf{R}^{\prime}\right)+\sigma_{y}(\mathbf{R}) \sigma_{y}\left(\mathbf{R}^{\prime}\right)+\sigma_{z}(\mathbf{R}) \sigma_{z}\left(\mathbf{R}^{\prime}\right)
$$

with $\sigma_{x}, \sigma_{y}$ as well as $\sigma_{s}$ being ordinary (as opposed to Pauli matrices) numerical vector components.

Magnetization is now defined (in accordance with the prescription from classical statistical mechanics) as a ratio of multiple integrals:

$m=\frac{\int \cdots \int \frac{1}{N}\left(\sum \sigma_{z}(\mathrm{R})\right) \exp \left\{\beta \sum J\left(\mathrm{R}-\mathrm{R}^{\prime}\right) \boldsymbol{\sigma}(\mathrm{R}) \cdot \boldsymbol{\sigma}\left(\mathrm{R}^{\prime}\right)+\omega \sum \sigma_{z}(\mathrm{R})\right\} d \mathrm{O}_{1} \cdots d \mathrm{O}_{N}}{\int \cdots \int \exp \left\{\beta \sum J\left(\mathrm{R}-\mathrm{R}^{\prime}\right) \boldsymbol{\delta}(\mathrm{R}) \cdot \boldsymbol{\sigma}\left(\mathrm{R}^{\prime}\right)+\omega \sum \sigma_{z}(\mathrm{R})\right\} d \mathrm{O}_{1} \cdots d \mathrm{O}_{N}}$

where each "single" integral is over the surface of a three-dimensional unit sphere, $d \mathrm{O}_{1}, d \mathrm{O}_{2}, \cdots$ being the surface elements of integration.

Again, as $N \rightarrow \infty$ we obtain $m^{*}(\omega ; \beta)$ (although I am not aware of anyone having explicitly proved the existence of the limit) and the problem of residual magnetization can again be posed.

For a two-dimensional lattice and for sufficiently short-ranged interactions, as expressed by the convergence of the series $\sum J(\mathbf{R})|\mathbf{R}|^{2}$ taken over the infinite lattice, Mermin [4] showed that again there is no residual magnetization at any temperature. 
It is generally believed that for three-dimensional lattices residual magnetization is present for sufficiently low temperatures, but nobody knows how to prove it.

It is this problem that I would like to offer as a test of whether a proposed reformulation of classical statistical mechanics has been more than an exercise. If it throws some non-trivial light on this problem I'll buy it and so will many others who have given serious thought to this problem. If not-no sale; for the reformulation is but another way to express one's ignorance.

9. Let me now suggest how some enlightenment may perhaps come.

In 1952 the late T. H. Berlin and I introduced a model which has become known as the spherical model. It is a mutilation of the Ising-Lenz model which consists in replacing the $\mu(R)$ 's which can assume only values \pm 1 by continuous variables $x(\mathbf{R})$ subject to a global constraint

$$
\sum_{\mathbf{R}} x^{2}(\mathrm{R})=N
$$

and replacing the sums by integrals over the sphere (9.1) (hence the "spherical" in the name of the model) with uniform density.

It is an artificial model but it has two great virtues: it is exactly soluble, and for short-ranged $J(\mathrm{R})$ (e.g. nearest-neighbor) it behaves with respect to dimensionality as do the Heisenberg models (classical and quantum-mechanical); i.e., it has no residual magnetization at any temperature for two-dimensional lattices and it is residually magnetized for sufficiently low temperatures for three-dimensional lattices.

The model proved to be a little more realistic than it had perhaps any right to be, and there is a huge literature dealing with it. But it was a rather recent discovery of Stanley [5] that may give the model a more fundamental status. What Stanley $^{2}$ observed was that if the classical three-dimensional "spins" $\mathbf{\sigma}(\mathbf{R})$ which are unit three-dimensional vectors are replaced by $d$-dimensional vectors of length $d$, then in the limit as $N \rightarrow \infty$ (thermodynamic limit) and then $d \rightarrow \infty$, one obtains the spherical model.

To be more precise, here is what one can actually prove. Let

$$
Q_{N}^{(d)}(\omega ; \beta)=\int \cdots \int \exp \left\{\beta d \sum J\left(\mathbf{R}-\mathbf{R}^{\prime}\right) \mathbf{\sigma}(\mathbf{R}) \cdot \mathbf{\sigma}\left(\mathbf{R}^{\prime}\right)+\omega d \sum \boldsymbol{\sigma}(\mathbf{R}) \cdot \boldsymbol{k}\right\} d \mathrm{O}_{1} \cdots d \mathrm{O}_{N}
$$

where each "single" integral is over the surface of a $d$-dimensional unit sphere and $\boldsymbol{x}$ is a fixed unit vector; then

$$
\lim _{d \rightarrow \infty, N \rightarrow \infty} \frac{1}{N d} \log Q_{N}^{(d)}=\lim _{N \rightarrow \infty} \frac{1}{N} \log Q_{N}^{(S)}
$$

with $Q_{N}^{(s)}$ denoting the partition function of the spherical model, i.e.

$$
Q_{N}^{(S)}=\int_{\sum_{x^{\prime}(\mathrm{R})-N}} \exp \left\{\beta \sum J\left(\mathrm{R}-\mathrm{R}^{\prime}\right) x(\mathrm{R}) x\left(\mathrm{R}^{\prime}\right)+\omega \sum x(\mathrm{R})\right\} d \mathrm{O}_{N} .
$$

$\left(d \mathrm{O}_{n}\right.$ is the surface element of the $N$-dimensional sphere of radius $\sqrt{ } N$.)

It is very likely that also

$$
\lim _{d \rightarrow \infty} m_{d}^{*}(\beta ; \omega)=m_{S}^{*}(\beta ; \omega),
$$

${ }^{2}$ Stanley's proof contained a doubtful point. A proof based on a different principle was recently supplied by Kac and Thompson (to appear). 
i.e.

$$
\lim _{d \rightarrow \infty} \lim _{N \rightarrow \infty} \frac{1}{N d} \frac{\partial}{\partial \omega} \log Q_{N}^{(d)}=\lim _{N \rightarrow \infty} \frac{1}{N} \frac{\partial}{\partial \omega} \log Q_{N}^{(S)},
$$

but I have no proof and it will probably be rather delicate.

What is however much more important (and without doubt much more difficult) is to prove that

$$
\frac{1}{d} \lim _{N \rightarrow \infty} \frac{1}{N} \frac{\partial}{\partial \omega} \log Q_{N}^{(d)}
$$

is, for fixed $\omega>0$, monotomically decreasing in $d$, or that at least

$$
\frac{1}{d} \lim _{N \rightarrow \infty} \frac{1}{N} \frac{\partial}{\partial \omega} \log Q_{N}^{(d)} \geq \lim _{N \rightarrow \infty} \frac{1}{N} \frac{\partial}{\partial \omega} \log Q_{N}^{(S)},
$$

indicating that the spherical model is the most reluctant to exhibit residual magnetization. $^{3}$

10. I would like to discuss briefly one more example which is meant to illustrate a different point. It concerns the old problem of the distribution of the eigenvalues of the Laplacian, and I thought that except for a number of interesting unsolved purely mathematical problems, there was not much new that was likely to come out.

That I was mistaken in this view became evident only a few weeks ago when, with Drs. Uhlenbeck and Putterman, I became involved in a discussion of an ideal BoseEinstein gas in a rotating bucket. Usually one simplifies one's life by taking a cylindrical bucket which requires here and there the use of rather special properties of Bessel functions. Well, Dr. Uhlenbeck proved to be more of a "pure" mathematician than I because he rightly felt that the use of special properties of Bessel functions should not be required, and he therefore suggested that we consider a general solid of revolution rather than just a cylinder. He then proposed that one should try to generalize the classical theorem of Weyl by considering not just the distribution of energies but, in a sense, the joint distribution of energies and angular momenta. Once the problem is posed, the solution becomes almost immediate.

Let the solid of revolution be obtained by revolving the curve $r=f(z) \geq 0,0 \leq z \leq L$, about the $z$-axis, and consider the eigenvalue problem

$$
\nabla^{2} U+E U=0
$$

with $U=0$ on the boundary of the solid of revolution.

The normalized eigenfunctions are of the form

$$
U_{l, p}(\mathbf{r})=\left(\exp (i l \theta) /(2 \pi)^{1 / 2}\right) u_{l, p}(r, z), \quad l=0, \pm 1, \pm 2, \cdots
$$

${ }^{3}$ However, one must be warned that this statement can only be true if the interaction $J\left(R, R^{\prime}\right)$ is not too anisotropic. As observed by Lieb and Thompson [6], a one-dimensional spherical model with $J_{12}=J, J_{i k}=0,(i, k) \neq(1,2)$, will exhibit residual magnetization at sufficiently low temperatures, while for no $d$ (including $d=1$, in which case the model is to be interpreted as the Ising-Lenz model) will this phenomenon occur. On the other hand, for such a pathological interaction, Stanley's theorem is not valid, and there are no grounds for comparing the $d$-dimensional models with the spherical one. 
(there is a trivial degeneracy) where

$$
\int_{0}^{L} d z \int_{0}^{f(z)} r u_{l, p}^{2}(r, z) d r=1
$$

We now consider the diffusion equation

$$
\partial P / \partial t=\nabla^{2} P
$$

with the surface of the solid of revolution an absorbing barrier, and we have for the Green's function $P\left(\mathbf{r} \mid \mathbf{r}^{\prime} ; t\right)$ the familiar formula

$$
\begin{aligned}
P\left(\mathbf{r} \mid \mathbf{r}^{\prime} ; t\right) & =\sum \exp \left(-t E_{l, p}\right) U_{l, p}(\mathbf{r}) U_{l, p}\left(\mathbf{r}^{\prime}\right) \\
& =(1 / 2 \pi) \sum \exp \left(-t E_{l, p}\right) \exp \left\{i l\left(\theta-\theta^{\prime}\right)\right\} u_{l, p}(r, z) u_{l, p}\left(r^{\prime}, z^{\prime}\right) .
\end{aligned}
$$

By the "principle of not feeling the boundary" [7] we have for small $t$

$(1 / 2 \pi) \sum \exp \left(-t E_{l, p}\right) \exp \left\{i l\left(\theta-\theta^{\prime}\right)\right\} u_{l, p}(r, z) u_{l, p}\left(r^{\prime}, z^{\prime}\right)$

$$
\begin{aligned}
& \sim \frac{1}{\left((4 \pi t)^{1 / 2}\right)^{3}} \exp \left\{-\frac{\left\|\mathbf{r}-\mathbf{r}^{\prime}\right\|^{2}}{4 t}\right\} \\
& =(4 \pi t)^{-3 / 2} \exp \left\{-\frac{r^{2}+r^{\prime 2}}{4 t}\right\} \exp \left\{-\frac{\left(z-z^{\prime}\right)^{2}}{4 t}\right\} \exp \left\{\frac{r r^{\prime}}{2 t} \cos \left(\theta-\theta^{\prime}\right)\right\},
\end{aligned}
$$

and now I take a leap by assuming that the above asymptotic relation holds even after differentiating it any number of times with respect to $\left(\theta-\theta^{\prime}\right)$. In other words, I assume that also

$$
\begin{aligned}
& \frac{1}{2 \pi} \sum \exp \left(-t E_{l, \mathrm{D}}\right)(i l)^{m} \exp \left\{i l\left(\theta-\theta^{\prime}\right)\right\} u_{l, \mathrm{p}}(r, z) u_{l, \mathrm{D}}\left(r^{\prime}, z^{\prime}\right) \\
& \sim(4 \pi t)^{-3 / 2} \exp \left\{-\frac{r^{2}+{r^{\prime 2}}^{2}}{4 t}\right\} \exp \left\{-\frac{\left(z-z^{\prime}\right)^{2}}{2 t}\right\} \frac{\partial^{m}}{\partial\left(\theta-\theta^{\prime}\right)^{m}} \exp \left\{\frac{r r^{\prime}}{2 t} \cos \left(\theta-\theta^{\prime}\right)\right\}
\end{aligned}
$$

for $m=1,2,3, \cdots$ and that moreover I can put $\mathbf{r}=\mathbf{r}^{\prime}$ (i.e., $\theta=\theta^{\prime}, r=r^{\prime}, z=z^{\prime}$ ) and integrate over the bucket.

Recalling that $t$ is small, all this leads, for $m=2 k$ (for odd $m$ the answer is 0 ), to the formula

$$
\sum \exp \left(-t E_{l, D}\right) l^{2 k} \sim \frac{(2 k) !}{2^{2 k} k !} \frac{1}{(4 \pi)^{3 / 2}}(2 \pi) \int_{0}^{L} d z \int_{0}^{f(z)} r^{2 k+1} d r t^{-(k+8 / 2)},
$$

where on the left $l$ runs from $-\infty$ to $\infty$.

While I do not have a proof, I have also no doubt that the asymptotic formula is correct, and I shall proceed on this assumption.

Let

$$
N^{(k)}(\lambda)=\sum_{E_{l, p<\lambda}} l^{2 k}
$$

then it follows by an application of a Tauberian theorem that

$$
N^{(k)}(\lambda) \sim\left(C(k) / \Gamma\left(k+\frac{5}{2}\right)\right) \lambda^{k+3 / 2}, \quad \lambda \rightarrow \infty,
$$

where 


$$
C(k)=\frac{(2 k) !}{2^{2 k} k !} \frac{1}{(4 \pi)^{3 / 2}} \frac{\pi}{k+1} \int_{0}^{L} f^{2(k+1)}(z) d z .
$$

Let now for a fixed $l$

$$
N_{l}(\lambda)=\sum_{E_{p}, l<\lambda} 1
$$

so that

$$
N^{k}(\lambda)=\sum_{l} l^{2 k} N_{l}(\lambda)=2 \sum_{l>0} l^{2 k} N_{l}(\lambda)
$$

The asymptotic formula for $N^{(k)}(\lambda)$ is now rewritten in the equivalent form:

$$
\lim _{\lambda \rightarrow \infty} \frac{1}{\lambda^{3 / 2}} \sum_{l>0}\left(\frac{l}{\sqrt{ } \lambda}\right)^{2(k+1)} \frac{1}{l^{2} / \lambda} N_{l}(\lambda)=\frac{1}{2}\left[\frac{C(k)}{\Gamma\left(k+\frac{5}{2}\right)}\right),
$$

and the crux of the matter is to notice that

$$
\frac{1}{2} \frac{C(k)}{\Gamma\left(k+\frac{5}{2}\right)}=\frac{1}{2} \frac{1}{2 \pi} \int_{0}^{1} \int_{0}^{L}(f(z) x)^{2(k+1)}\left(\frac{1}{x}-1\right)^{2} d z d x
$$

It now follows by a standard argument that

$$
\lim _{\lambda \rightarrow \infty} \frac{1}{\lambda^{3 / 2}} \sum_{0<\alpha<l / \sqrt{ } \lambda<\beta} \frac{2}{l^{2} / \lambda} N_{l}(\lambda)=\frac{1}{2} \frac{1}{2 \pi} \iint\left(\frac{1}{x}-1\right)^{2} d x,
$$

where the double integral is over the region in the $x, z$-plane defined by the inequalities $0<\alpha<x f(z)<\beta, 0 \leq x \leq 1,0 \leq z \leq L$.

This is one of the forms of the desired extension of Weyl's theorem, and it follows from it that

$$
\lim _{\lambda \rightarrow \infty} \frac{1}{\lambda^{3 / 2}} \sum_{0<\alpha<l / \sqrt{ } \lambda<\beta} \frac{1}{l^{2} / \lambda} N_{l}(\lambda)
$$

determines uniquely not quite $f(z)$ itself but the distribution of its values, i.e., the function

$$
\sigma_{f}(u)=\mu\{f(z)<u, 0 \leq z \leq L\},
$$

where $\mu\{\}$ denotes the Lebesgue measure of the set in braces.

However, as is well known, the eigenvalues allow one to determine the surface area of the solid and, in particular, its lateral surface area*

$$
2 \pi \int_{0}^{L} f(z)\left(1+\left(\frac{d f}{d z}\right)^{2}\right)^{1 / 2} d z
$$

It is clear now that if $\phi(z)$ is a piecewise-differentiable measure-preserving transformation of the interval $(0, L)$ onto itself, then the solids of revolution generated by $f(z)$ and by $f(\phi(z))$ will have different lateral surface areas unless $\phi(z) \equiv z$. In other words, if two solids of revolution have the same eigenvalues, they are congruent.

* Added in proof: This is unfortunately not so and I must assume that I also know the area of the circular bases. 
Now, the result (10.1) and what it implies is without doubt of interest apart from the particular application which led to it. It belongs to "pure" mathematics just as much as it belongs to "applied". What struck me was that in the vast literature generated by Weyl's theorem there seems to be no hint that anyone has ever considered the extension which I discussed.

Solids of revolution are, of course, rather special, and there would be no a priori reason to expect that anything of "general" interest would be discovered by studying their eigenvalues. It is only because the problem of rotating Bose-Einstein fluids is of great physical interest (because of helium) that containers which are solids of revolution become worthy of study. And, as happens over and over again when one applies mathematics to genuine problems, one reaps unexpected harvests.

11. A final reflection. The models with $d$-dimensional spins as well as the spherical model have all the earmarks of being "dehydrated elephants", and it may turn out that indeed they are. In inventing and discussing such models, we are applying mathematics in a way quite different from the more traditional ones about which we heard so much during this conference and which is also illustrated by the example of Sec. 10. But what gives these models a hold on life - tenuous though this hold may be-is that in spite of admitted lack of realism, they are firmly rooted in reality, and they were conceived to deal with real questions. Without such rooting and without real questions to guide us, we may well find ourselves fighting windmills and triumphantly emerging with pyrrhic victories.

Appendix. In this appendix I reproduce Mermin's proof [4] that $m^{*}=0$ for all temperatures for a two-dimensional square lattice and planar "spins" $(d=2)$. I do it to show that in the right hands, Schwarz's inequality and integration by parts are still among the most powerful tools of analysis.

In the case under consideration we have

$$
H=-\sum_{\mathbf{R}, \mathbf{R}^{\prime}} J\left(\left|\mathbf{R}-\mathbf{R}^{\prime}\right|\right) \cos \left(\theta(\mathbf{R})-\theta\left(\mathbf{R}^{\prime}\right)\right)-a \sum_{\mathbf{R}} \cos \theta(\mathbf{R}),
$$

and we introduce two auxiliary expressions

$$
A=\sum_{\mathbf{R}} \exp (-i \mathbf{k} \cdot \mathrm{R}) \sin \theta(\mathrm{R})
$$

and

$$
B=\sum_{\mathbf{R}} \exp (-i \mathbf{k} \cdot \mathbf{R})(\partial H / \partial \theta(\mathbf{R})) .
$$

We shall denote by \langle\rangle averages with respect to the canonical weight $\exp (-\beta H)$; i.e., for a function $F$ of the angles $\theta(\mathbf{R})(0 \leq \theta(\mathbf{R})<2 \pi)$, we have

$$
\langle F\rangle=\frac{\int_{0}^{2 \pi} \cdots \int_{0}^{2 \pi} F \exp \{-\beta H\} \prod_{\mathbf{R}} d \theta(\mathrm{R})}{\int_{0}^{2 \pi} \cdots \int_{0}^{2 \pi} \exp \{-\beta H\} \prod_{\mathbf{R}} d \theta(\mathbf{R})} .
$$

By Schwarz's inequality we have

$$
\left\langle A A^{*}\right\rangle \geq\left|\left\langle A B^{*}\right\rangle\right|^{2} /\left\langle B B^{*}\right\rangle .
$$


Now,

$$
B B^{*}=\sum_{\mathbf{R}, \mathbf{R}^{\prime}} \exp \left\{-i \mathbf{k} \cdot\left(\mathbf{R}-\mathbf{R}^{\prime}\right)\right\} \frac{\partial H}{\partial \theta(\mathbf{R})} \frac{\partial H}{\partial \theta\left(\mathbf{R}^{\prime}\right)}
$$

and since

$$
\frac{\partial H}{\partial \theta\left(\mathbf{R}^{\prime}\right)} \exp \{-\beta H\}=-\frac{1}{\beta} \frac{\partial}{\partial \theta\left(\mathbf{R}^{\prime}\right)} \exp \{-\beta H\},
$$

we get, by integration by parts,

$$
\begin{aligned}
& \left\langle B B^{*}\right\rangle=\frac{1}{\beta}\left\langle\sum_{\mathbf{R}, \mathbf{R}^{\prime}} \exp \left\{i \mathbf{k} \cdot\left(\mathbf{R}-\mathbf{R}^{\prime}\right)\right\} \frac{\partial^{2} H}{\partial \theta(\mathbf{R}) \partial \theta\left(\mathbf{R}^{\prime}\right)}\right\rangle \\
& =\frac{1}{\beta} \sum_{\mathbf{R}, \mathbf{R}^{\prime}} J\left(\left|\mathbf{R}-\mathbf{R}^{\prime}\right|\right)\left(1-\exp \left\{i \mathbf{k} \cdot\left(\mathbf{R}-\mathbf{R}^{\prime}\right)\right\}\right)\left\langle\cos \left(\theta(\mathbf{R})-\theta\left(\mathbf{R}^{\prime}\right)\right)\right\rangle-\frac{a}{\beta} \sum_{\mathbf{R}}\langle\cos \theta(\mathbf{R})\rangle,
\end{aligned}
$$

where we have used the obvious identity

$$
\sum_{\mathbf{R}, \mathbf{R}^{\prime}} J\left(\left|\mathbf{R}-\mathbf{R}^{\prime}\right|\right)\left\langle\cos \left(\theta(\mathbf{R})-\theta\left(\mathbf{R}^{\prime}\right)\right\rangle=0 .\right.
$$

Since $\left\langle B B^{*}\right\rangle$ is clearly real and since

$$
\sum_{\mathbf{R}}\langle\cos \theta(\mathbf{R})\rangle=N m,
$$

where $m$ is the magnetization, we have

$$
\left\langle B B^{*}\right\rangle=\frac{1}{\beta} \sum J\left(\left|\mathbf{R}-\mathbf{R}^{\prime}\right|\right)\left[1-\cos \mathbf{k} \cdot\left(\mathbf{R}-\mathbf{R}^{\prime}\right)\right]\left\langle\cos \left(\theta(\mathbf{R})-\theta\left(\mathbf{R}^{\prime}\right)\right)\right\rangle-\frac{a N m}{\beta},
$$

and hence

$$
\left\langle B B^{*}\right\rangle \leq \frac{1}{2 \beta}|\mathbf{k}|^{2} \sum_{\mathbf{R}, \mathbf{R}^{\prime}} J\left(\left|\mathbf{R}-\mathbf{R}^{\prime}\right|\right)\left|\mathbf{R}-\mathbf{R}^{\prime}\right|^{2}+\frac{|a| N|m|}{\beta} .
$$

Since clearly

$$
\sum J\left(\left|\mathbf{R}-\mathbf{R}^{\prime}\right|\right)\left|\mathbf{R}-\mathbf{R}^{\prime}\right|^{2} \leq N \sum J(R) R^{2},
$$

we have finally

$$
\left\langle B B^{*}\right\rangle \leq \frac{N}{\beta}\left\{\alpha|\mathbf{k}|^{2}+|a||m|\right\},
$$

where

$$
\alpha=\frac{1}{2} \sum J(R) R^{2}
$$

Furthermore,

$$
A^{*} B=\sum_{\mathbf{R}, \mathbf{R}^{\prime}} \exp \left\{\imath \mathrm{k} \cdot\left(\mathrm{R}, \mathrm{R}^{\prime}\right)\right\} \sin \theta(\mathrm{R}) \frac{\partial H}{\partial \theta\left(\mathbf{R}^{\prime}\right)}
$$

and in integrating $A^{*} B \exp \{-\beta H\}$ we see that only terms with $\mathbf{R}=\mathbf{R}^{\prime}$ survive. Inte- 
gration by parts finally gives

$$
\left|\left\langle A^{*} B\right\rangle\right|=\frac{1}{\beta} N|m|
$$

and, combining this with (A.6) and (A.5), we obtain

$$
\left\langle A A^{*}\right\rangle \geq \frac{N|m|^{2}}{\alpha|\mathrm{k}|^{2}+|a||m|} .
$$

Since

$$
\left\langle A A^{*}\right\rangle=\sum_{\mathbf{R}, \mathbf{R}^{\prime}}\left\langle\sin \theta(\mathbf{R}) \sin \theta\left(\mathbf{R}^{\prime}\right)\right\rangle \exp \left\{i \mathbf{k} \cdot\left(\mathbf{R}-\mathbf{R}^{\prime}\right)\right\},
$$

we write (A.9) in the equivalent form

$$
\sum_{\mathbf{R}, \mathbf{R}^{\prime}}\left\langle\sin \theta(\mathbf{R}) \sin \theta\left(\mathbf{R}^{\prime}\right)\right\rangle \exp \left\{i \mathbf{k} \cdot\left(\mathbf{R}-\mathbf{R}^{\prime}\right)\right\} \geq \frac{N m^{2}}{\alpha|\mathbf{k}|^{2}+|a||m|}
$$

The inequality (A.10) is valid for every two-dimensional vector $\mathbf{k}$ and the idea is to sum both sides of (A.10) over an appropriately chosen set of k's.

For the sake of simplicity and definiteness, let

$$
\mathbf{R}=l \mathbf{l}_{1}+m \mathbf{l}_{\mathbf{2}}, \quad \mathbf{R}_{1}=l^{\prime} \mathbf{1}_{1}+m^{\prime} \mathbf{1}_{\mathbf{2}}
$$

with $l_{1}$ and $l_{2}$ mutually orthogonal unit vectors and $l, m, l^{\prime}, m^{\prime}$ integers. Let also $N$ be a perfect square and set

$$
\mathbf{k}=(2 \pi / \sqrt{ } N)\left(p \mathbf{l}_{1}+q \mathbf{l}_{2}\right),
$$

where $p, q$ are integers running from 0 to $\sqrt{ } N-1$ ( $\sqrt{ } N$ is assumed to be an integer).

With $\mathbf{k}$ running through the set (A.11) we have

$$
\begin{aligned}
\sum_{\mathbf{k}} \exp \left\{\imath \mathbf{k} \cdot\left(\mathrm{R}-\mathrm{R}^{\prime}\right)\right\} & =N, & & \mathbf{R}=\mathrm{R}^{\prime} \\
& =0, & & \mathbf{R} \neq \mathbf{R}^{\prime}
\end{aligned}
$$

and hence

$$
\sum_{\mathbf{R}}\left\langle\sin ^{2} \theta(\mathbf{R})\right\rangle \geq m^{2} \sum_{\mathbf{k}} \frac{1}{\alpha|\mathbf{k}|^{2}+|a||m|} .
$$

Since $\left\langle\sin ^{2} \theta(\mathbf{R})\right\rangle \leq 1$, we have

$$
1 \geq m^{2} \frac{1}{N} \sum_{\mathbf{k}} \frac{1}{\alpha|\mathbf{k}|^{2}+|a||m|}
$$

which in the limit $N \rightarrow \infty$ becomes

$$
1 \geq \frac{m^{*^{2}}}{(2 \pi)^{2}} \int_{0}^{1} \int_{0}^{1} \frac{d x d y}{\alpha\left(x^{2}+y^{2}\right)+|a||m|^{*}} .
$$

Since

$$
\int_{0}^{1} \int_{0}^{1} d x d y /\left(x^{2}+y^{2}\right)=\infty
$$

it follows at once that $\lim _{a \rightarrow 0} m^{*}=0$ and the proof is complete. 


\section{REFERENCES}

[1] Saunders MacLane, Hamiltonian mechanics and geometry, Am. Math. Monthly 77, 570-586 (1970)

[2] Joel E. Cohen, Mathematics as metaphor, Science 172, 674-675 (1971)

[3] N. D. Mermin and H. Wagner, Absence of ferromagnetism or antiferromagnetism in one- or twodimensional isotropic Heisenberg models, Phys. Rev. Letters 17, 1133-1136 (1960)

[4] N. D. Mermin, Absence of ordering on certain classical systems, J. Math. Phys. 8, 1061-1064 (1967)

[5] H. E. Stanley, Spherical model as the limit of infinite spin dimensionality, Phys. Rev. 176, 718-722 (1968)

[6] E. H. Lieb and C. J. Thompson, Phase transition in zero dimensions: a remark on the spherical model, J. Math. Phys. 10, 1403-1406 (1969)

[7] M. Kac, Can one hear the shape of a drum?, Am. Math. Monthly 73, 1-23 (1966) 